6. Anz $D$, et al. Suppression of intratumoral CCL22 by type $i$ interferon inhibits migration of regulatory $T$ cells and blocks cancer progression. Cancer Res 2015;75(21):4483-93

Disclosure Information J. Grün: None. I. Piseddu: None. C. Perleberg: None. N. Röhrle: None. S. Endres: None. D. Anz: None.

\section{P01.03 TARGETING DIACLYGLYCEROL KINASE ALPHA AND ZETA BY SELF DELIVERING RNAI TO OPTIMIZE TLYMPHOCYTES FOR ADOPTIVE THERAPY OF SOLID TUMORS}

${ }^{1}$ AS Herbstritt", ${ }^{2} \mathrm{M}$ Maxwell, ${ }^{2} \mathrm{D}$ Yan, ${ }^{2} \mathrm{~B}$ Cuiffo, ${ }^{2} \mathrm{~J}$ Cardia, ${ }^{2} \mathrm{SP}$ Fricker, ${ }^{1} \mathrm{E}$ Noessner. ${ }^{1}$ Helmholtz Zentrum München, Munich, Germany; ${ }^{2}$ Phio Pharmaceuticals, Marlborough, MA, USA

\subsection{6/jitc-2021-ITOC8.7}

Background Evidence indicates that diacylglycerol kinases (DGK) are promising targets for the optimization of $\mathrm{T}$ cell activity, for example in the setting of adoptive cell therapy (ACT). The tumor microenvironment (TME) of human renal cell carcinoma (RCC) is an immunosuppressive setting where $\mathrm{T}$ and NK cell functionality is blocked. DGK- $\alpha$ is a negative regulator of TCR signaling, functioning by metabolizing diacylglycerol to phosphatidic acid and thereby limiting the activation of MAPK/ERK1/2 signaling pathway. DGK- $\alpha$ is found increased in tumor-infiltrating lymphocytes (TIL) from RCC patients and also in adoptively transferred $\mathrm{T}$ cells after infiltrating into the TME. ${ }^{1}$ We previously reported that inhibition of DGK- $\alpha$ restored functionality of unresponsive CD8 T cells and NK cells from RCC-TIL. Other studies demonstrated that knockdown or pharmacologic inhibition of DGK- $\alpha$ and DGK$\zeta$ alone or together increased target cell killing and cytokine production, and protected $\mathrm{T}$ cells from inhibitory factors in the TME. ${ }^{2}$ However, there are no inhibitors for DGK- $\zeta$ and available DGK- $\alpha$ inhibitors have undesired pharmacokinetic/ pharmacodynamic properties and are highly toxic precluding their clinical application. Here, we present data using a novel RNA interference (RNAi) technology that can specifically target each DGK isoform.

Materials and Methods INTASYL ${ }^{\mathrm{TM}}$ compounds incorporate drug-like properties into RNAi, resulting not only in enhanced cellular uptake in the presence of serum but also eliminating the need for further transfection reagents. Toxicity of compounds applied alone or in combination was assessed by 7-AAD flow cytometry analysis and WST assay. Silencing of mRNA and protein was analyzed by RT-qPCR and SimpleWestern. Downstream signaling pathways and $\mathrm{T}$ cell function were analyzed to demonstrate pharmacological efficacy.

Results Two DGK- $\zeta$ compounds and one DGK- $\alpha$ compound were analyzed using Jurkat $\mathrm{T}$ cells and primary human TCRtransduced $\mathrm{T}$ cells. No effects were seen on cell viability for the compounds applied alone or in combination. On-target knockdown was achieved in Jurkat $\mathrm{T}$ cells evidenced by RTqPCR and SimpleWestern. Silencing of mRNA and protein occurred quickly after $24 \mathrm{~h}$, peaked between $48 \mathrm{~h}$ and $72 \mathrm{~h}$ and lasted at least for 96h. Stimulation under DGK-targeting INTASYL treatment resulted in enhanced levels of phosphorylated ERK1/2 and enhanced secretion of IL-2.

Conclusions INTASYL ${ }^{\mathrm{TM}}$ self-delivering RNAi compounds represent a promising approach to target intracellular immune checkpoints such as DGKs. The good toxicity profile allows for combined application of several compounds enabling targeting of multiple checkpoints, which likely is necessary to counteract the complex and heterogeneous inhibitory influences of the TME. The technology enables the anti-tumor activity of $\mathrm{T}$ and $\mathrm{NK}$ cells for immunotherapy, and can be used in ACT and direct therapeutic applications towards the TME.

\section{REFERENCES}

1. Moon EK, Wang L-C, Dolfi DV, Wilson CB, Ranganathan R, Sun J, et al. Multifactorial T-cell hypofunction that is reversible can limit the efficacy of chimeric antigen receptor-transduced human T cells in solid tumors. Clin Cancer Res 2014;20 (16): $4262-73$

2. Jung I-Y, Kim Y-Y, Yu H-S, Lee M, Kim S, Lee J. CRISPR/Cas9-mediated knockout of DGK improves antitumor activities of human T cells. Cancer Res 2018:78 (16):4692-703.

Disclosure Information A.S. Herbstritt: C. Other Research Support (supplies, equipment, receipt of drugs or other in-kind support); Significant; Phio Pharmaceuticals. M. Maxwell: A. Employment (full or part-time); Significant; Phio Pharmaceuticals. D. Yan: A. Employment (full or part-time); Significant; Phio Pharmaceuticals. B. Cuiffo: A. Employment (full or parttime); Significant; Phio Pharmaceuticals. J. Cardia: A. Employment (full or part-time); Significant; Phio Pharmaceuticals. S.P. Fricker: A. Employment (full or part-time); Significant; Phio Pharmaceuticals. E. Noessner: C. Other Research Support (supplies, equipment, receipt of drugs or other in-kind support); Significant; Phio Pharmaceuticals.

\section{P01.04 LENTIVIRAL PROTEIN VPX DELIVERY SYSTEMS AS POTENTIAL WEAPONS TO IMPROVE CYTARABINE TREATMENT RESPONSE AGAINST ACUTE MYELOID LEUKEMIA}

R Nair* , H Baldauf. Max von Pettenkofer Institute, Munich, Germany

\subsection{6/jitc-2021-ITOC8.8}

Background Acute myeloid leukemia (AML) is an aggressive cancer of the blood, where malignant myeloid blasts accumulate in the bone marrow. One of the challenges of effective AML treatment is resistance to cytarabine (or ara-C), a standard AML chemotherapeutic drug used in front-line treatment today. In 2017, Schneider et al. reported the dNTPase sterile alpha motif and HD-domain-containing protein 1 (SAMHD1) to be a targetable biomarker for ara-C treatment response. ${ }^{1}$ The intracellular triphosphorylated active form of ara-C, araCTP, was recognized as a substrate by SAMHD1 and is hydrolyzed back to ara-C. This led to a decrease in the amount of ara-CTP within the cells and consequently reduced cytotoxicity. ${ }^{1}$ SAMHD1 can be targeted by the lentiviral accessory protein $\mathrm{Vpx}$ for proteasomal degradation by interacting with the proteasomal degradation complex and SAMHD1. This study aims to use Vpx to target SAMHD1 in AML cells to improve ara-C sensitivity.

Materials and Methods In order to manipulate SAMDH1 levels using Vpx, different Vpx delivery systems were developed. These are virus-like particles (VLPs) packaged with different homologs of $\mathrm{Vpx}$ from Simian Immunodeficiency Viruses (SIV) and HIV-2, and cell-penetrating peptides (CPPs) bound to either a 67 amino acid truncated SIVmac Vpx (67aaVpx) or to the WT full-length form. Two different CPPs were used in the synthesis: TAT and CPP44. The latter 